positive) and in 34 - in CMA. IgM-aß2GP1 was combined with IgA-aß2GP1 with the same frequency in both methods (in 13 patients).

IgA-aß2GP1 was detected in $30(48 \%)$ of 63 patients. They were combined with both IgG-aCL and IgG-aß2GP1 in all cases in both methods. IgM-aCL and IgMaß2GP1 were detected in 14 and 11 of 30 patients with IgA-aß2GP1, respectively. The combination of IgA-aß2GP1 with IgG-aCL by ELISA was in 27 (in most cases highly positive - 20) and with IgM-aCL - in 10 (highly positive - 8). IgG-aß2GP1 was detected in 28 patients with IgA-aß2GP1 (high positive - 21) and in 11 patients with IgM-aß2GP1 (high positive -7).

IgG-aß2GP1-D1 was revealed in 48 (76\%) patients. It was combined with IgG$\mathrm{aCL}$ - in 38, with IgM-aCL - in 15 patients by the ELISA. The combination of IgG-aß2GP1-D1 by CMA was as follows: with IgG-aCL - in 46, with IgM-aCL - in 17 , and with IgA-aCL - in 33 patients. In most cases, IgG-aß2gp1-D1 was combined with highly positive aCL levels. IgG-aß2GP1-D1 positivity was associated with IgG-aß2GP1 positivity in 42 - by ELISA and 47 - by CMA, IgM-aß2GP1 - in 13 and 14 patients by ELISA and CMA, respectively, and IgA-aß2GP1 - in 29. Isolated IgG-aß2GP1-D1 positivity was not observed.

Conclusion: The frequency of $\operatorname{lgA}-\mathrm{aCL}$ detection was $56 \%$ (35 patients out of 63 ), IgA-aß2GP1 - 48\% (30 patients out of 63 ), IgG-aß2GP1-D1 - 76\% (48 patients out of 63 ). There was not isolated positivity of this "extra" criterial antibodies. The presence of IgA-aCL, IgA-aß2GP1, IgG-aß2GP1-D1 was associated with highly positivity of $\lg \mathrm{G} / \operatorname{lgM}-\mathrm{aCL}$ and $\lg \mathrm{G} / \mathrm{lgM}-\mathrm{a} \beta 2 \mathrm{GP} 1$.

Disclosure of Interests: None declared

DOI: 10.1136/annrheumdis-2021-eular.818

\section{POS0713 PREDICTORS OF HOSPITALIZATION IN PATIENTS WITH SYSTEMIC LUPUS ERYTHEMATOSUS: A 10-YEAR COHORT STUDY OF 398 PATIENTS FROM A TERTIARY CENTRE}

H. Assunção $^{1}$, M. Rodrigues ${ }^{2}$, A. R. Prata3, J. A. P. Da Silva ${ }^{1,4}$, L. Inês ${ }^{1,5}$. ${ }^{1}$ Centro Hospitalar e Universitário de Coimbra, Rheumatology, Coimbra, Portugal; ${ }^{2}$ Hospital Distrital de Leiria, Rheumatology, Leiria, Portugal; ${ }^{1}$ Centro Hospitalar e Universitário de Coimbra, Rheumatology, Coimbra, Portugal; ${ }^{4}$ University of Coimbra, Coimbra Institute for Clinical and Biomedical Research (iCBR), Faculty of Medicine, Coimbra, Portugal; ${ }^{5}$ University of Beira Interior, School of Health Sciences, Covilhã, Portugal

Background: Patients with systemic lupus erythematosus (SLE) often require hospitalization. The cause of admission may vary, but active disease and infection are consistently reported as the main reasons for hospitalization and are associated with worse survival and damage accrual. Recent improvements in the standard of care, including minimization of glucocorticoid dose and more effective and safe immunosuppressive regimens, may have changed the incidence and risk factors for hospitalization due to these causes. Hence, it is useful to identify predictors of hospitalization to further reduce the risk of admission for disease activity and severe infection in patients with SLE.

Objectives: To identify predictors of hospitalization in patients with SLE, according to the underlying cause.

Methods: Patients with SLE fulfilling classification criteria (ACR'97 and/or SLICC), regularly followed at an academic lupus clinic from January 2009 to December 2020 and with at least two outpatient visits were included in this cohort study. Time to first hospitalization up to 120 months was identified separately for the following admission causes: (a) any cause; (b) active SLE; (c) infection. Predictors of hospitalization were sought through survival analysis, with distinct models for each of the major admission causes. Univariate analysis was performed using Kaplan-Meier curves and Log-Rank tests. Tested variables assessed at baseline included: gender; age at SLE onset; age; disease duration; SLE Disease Activity Index (SLEDAI-2K) score; ongoing antimalarial use; ongoing immunosuppressants; ongoing prednisoIone daily dose; lupus nephritis up to baseline; SLICC Damage Index (SDI) score. Variables with $p<0.1$ were further tested in multivariate Cox regression models. Hazard ratios (HR) were determined with $95 \%$ confidence intervals $(95 \% \mathrm{Cl})$.

Results: We included 398 patients (female: $86.2 \%$, mean age: $41.2 \pm 15.1$ years, mean disease duration: $10.1 \pm 9.2$ years; previous lupus nephritis: $28.9 \%$; mean SLEDAI-2K score: $3.4 \pm 2.7$; ongoing antimalarials: $78.9 \%$; ongoing immunosuppressant: $29.9 \%$; ongoing prednisolone $>7.5 \mathrm{mg} /$ day: $17.1 \%$; SDI score $\geq 1: 28.4 \%$ ). During the follow-up period, $50.5 \%, 23.6 \%$ and $17.3 \%$ were hospitalized at least once for any cause, active SLE or infection, respectively.

In the multivariate model, significant baseline predictors for hospitalization due to active disease were (table 1): SLEDAI-2K score $>5$; disease duration $\leq 2$ years; ongoing immunosuppressants; SDI score $\geq 1$. Baseline independent predictors of hospitalization for infection included (table 1): male gender; SDI score $\geq 1$; ongoing antimalarials were protective.

Table 1. Predictors of hospitalization in multivariate Cox regression according to the admission cause

\begin{tabular}{lcc}
\hline Predictors & Hospitalization for active SLE & Hospitalization for infection \\
\hline SLEDAl-2K score $>5$ & $2.43(1.53-3.88)$ & $n . s$. \\
SLE duration $\leq 2$ years & $1.70(1.04-2.77)$ & n.s. \\
Ongoing immunosuppressant & $1.91(1.24-2.95)$ & n.s. \\
SDI score $\geq 1$ & $1.82(1.16-2.86)$ & $2.14(1.33-3.45)$ \\
Male gender & n.s. & $2.19(1.23-3.89)$ \\
No antimalarial treatment & n.s. & $2.20(1.34-3.60)$
\end{tabular}

Risk for each predictor reported as Hazard Ratio (95\% Confidence Interval); n.s.: non-significan

Conclusion: Tight control of disease activity, prevention of damage accrual, and treatment with antimalarials may contribute to minimize the risk of hospitalization for these two major causes of admission in patients with SLE.

Disclosure of Interests: None declared

DOI: 10.1136/annrheumdis-2021-eular.832

\begin{tabular}{|l|l}
\hline POS0714 & WHITE MATTER HYPERINTENSITIES LEAD TO \\
REDUCED PSYCHOMOTOR SPEED IN PATIENTS \\
WITH SYSTEMIC LUPUS ERYTHEMATOSUS AND \\
NEUROPSYCHIATRIC SYMPTOMS
\end{tabular}

R. Monahan ${ }^{1}$, F. Inglese ${ }^{2}$, H. Middelkoop ${ }^{3}$, M. Van Buchem ${ }^{2}$, T. Huizinga ${ }^{1}$ M. Kloppenburg, ${ }^{1,4}$, I. Ronen ${ }^{2}$, G. M. Steup-Beekman ${ }^{1}$, J. De Bresser ${ }^{2} .{ }^{1}$ Leiden University Medical Center, Department of Rheumatology, Leiden, Netherlands; ${ }^{2}$ Leiden University Medical Center, Department of Radiology, Leiden, Netherlands; ' ${ }^{2}$ Leiden University Medical Center, Department of Neurology, Leiden, Netherlands; ${ }^{4}$ Leiden University Medical Center, Department of Clinical Epidemiology, Leiden, Netherlands

Background: Cognitive impairment is common in patients with systemic lupus erythematosus (SLE) and neuropsychiatric (NP) symptoms, but the exact underlying structural brain correlates are unknown.

Objectives: We aimed to compare cognitive function between groups of patients with different phenotypes of (NP)SLE and assessed the association between brain volumes, white matter hyperintensity $(\mathrm{WMH})$ volume and cognitive function. Methods: Patients who visited the NPSLE clinic of the Leiden University Medical Center between 2007-2015 were included in this study ( $n=151 ; 42 \pm 13$ years, $91 \%$ female). In a multidisciplinary consensus meeting, phenotypes were established and neuropsychiatric symptoms were attributed to SLE (NPSLE, inflammatory $(n=24)$ or ischemic $(n=12))$ or to minor involvement of SLE or other causes (minor/non-NPSLE $(n=115)$ ). All patients underwent standardized cognitive assessment of the four cognitive domains: global cognitive functioning (GCF), learning and memory (LM), executive functioning and complex attention (EFCA) and psychomotor speed (PS)). Cognitive dysfunction was defined as a T-score $<40$, with age, sex, gender and education matched individuals of the Dutch population as reference. In addition, automated volume measurements on brain MRI (white matter, grey matter, white matter hyperintensities (WMH) and total brain volume (TBV)) were performed. Patients with brain infarcts $>1.5 \mathrm{~cm}$ were excluded. Cognitive function (Z-score) was compared between different NPSLE phenotypes using multiple regression analyses corrected for age, sex and education. Associations between brain volumes, WMH and cognitive function were assessed per phenotype using multiple regression analyses corrected for age, sex and intracranial volume.

Results: Global cognitive functioning was impaired in $5 \%$, learning \& memory in $46 \%$, and executive functioning \& complex attention in $39 \%$ and psychomotor speed in $46 \%$ of all patients. Patients with inflammatory NPSLE showed the most cognitive impairment and reduced cognitive function compared to ischemic NPSLE (all domains) and minor/non-NPSLE (EFCA) ( $p$ $<0.05)$

Lower total brain volume and grey matter volume were associated with lower cognitive functioning in all domains $(\beta: 0.00 / 0.01(0.00 ; 0.01))$ and lower white matter volume associated with lower LM, EFCA and PS $(\beta: 0.00 / 0.01(0.00 ; 0.01)$ ) in all patients. Higher WMH volume associated with lower psychomotor speed $(\beta:-0.14(-0.32 ;-0.02))$, especially in patients with inflammatory NPSLE ( $\beta:-0.36$ $(-0.60 ;-0.12)$

Conclusion: Reduced brain volume leads to reduced cognitive function in multiple cognitive domains in all patients with SLE and neuropsychiatric symptoms. Increased WMH volume leads to reduced psychomotor speed, especially in patients with inflammatory NPSLE. 
Table 1. Prevalence of cognitive impairment in patients with minor/nonNPSLE, inflammatory NPSLE and ischemic NPSLE

\begin{tabular}{lcccc}
\hline & $\begin{array}{c}\text { Global cognitive } \\
\text { functioning }\end{array}$ & $\begin{array}{c}\text { Learning } \\
\text { memory }\end{array}$ & $\begin{array}{c}\text { Executive functioning \& } \\
\text { complex attention }\end{array}$ & $\begin{array}{c}\text { Psychomotor } \\
\text { speed }\end{array}$ \\
\hline All patients & $8(5)$ & $70(46)$ & $57(39)$ & $69(46)$ \\
$\begin{array}{l}(n=151) \\
\text { Inflammatory } \\
\text { NPSLE }\end{array}$ & $3(13)$ & $14(58)$ & $12(50)$ & $12(50)$ \\
$\begin{array}{l}\text { (n=24) } \\
\text { Ischemic NPSLE }\end{array}$ & $0(0)$ & $6(50)$ & $2(17)$ & $2(17)$ \\
$\begin{array}{l}(n=12) \\
\text { Minor/non-NPSLE }\end{array}$ & $5(4)$ & $50(44)$ & $43(38)$ & $55(49)$ \\
(n=115) & & & & \\
\hline
\end{tabular}

NPSLE = neuropsychiatric systemic lupus erythematosus.Data represent $\mathrm{n}(\%)$ of patients with cognitive impairment in the mentioned cognitive domain. Cognitive impairment was defined as cognitive function at least 1SD lower than the mean of an age, sex and education matched general Dutch population. The percentages were calculated from total number of patients with available scores: Global functioning: 23/24 inflammatory NPSLE and 113/115 minor/non-NPSLE; psychomotor speed 113/115 minor/non-NPSLE, executive function \& complex attention: also 113/115. All tests were available for ischemic NPSLE.

Disclosure of Interests: None declared

DOI: 10.1136/annrheumdis-2021-eular.881

\section{POS0715 $\quad$ QUANTIFYING THE PSYCHOSOCIAL IMPACT OF QUANTIFYING THE PSYCHOSOCIAL IMPACT
UNDIFFERENTIATED CONNECTIVE TISSUE DISEASE (UCTD)}

C. Siegel ${ }^{1,2}$, E. Sevim ${ }^{3,4}$, B. Stamm ${ }^{5}$, J. Vega ${ }^{5}$, J. Kleinman ${ }^{6}$, J. Barnhill ${ }^{7}$, M. Lockshin ${ }^{2,5}$, L. Sammaritano ${ }^{2,5}$, M. Barbhaiya ${ }^{2,5} .{ }^{1}$ Hospital for Special Surgery, Rheumatology, New York, United States of America; ${ }^{2}$ Weill Cornell Medicine, Internal Medicine, New York, United States of America; ${ }^{3}$ Montefiore Medical Center, Internal Medicine, Bronx, United States of America; ${ }^{4}$ Albert Einstein College of Medicine, Internal Medicine, Bronx, United States of America; ${ }^{5}$ Hospital for Special Surgery, Barbara Volcker Center for Women and Rheumatic Diseases, New York, United States of America; ${ }^{6}$ Hospital for Special Surgery, Department of Social Work Programs, Division of Rheumatology, New York, United States of America; ${ }^{7}$ Weill Cornell Medicine, Psychiatry, New York, United States of America

Background: Only half of patients diagnosed with SLE fulfill classification criteria; the rest have "SLE-like" illnesses such as UCTD. SLE patients are known to experience impaired health-related quality of life (HRQoL) and significant anxiety, depression, and fatigue, ${ }^{1}$ yet the psychosocial aspects of UCTD are less established. In a qualitative study, we found that most UCTD patients had engaged in psychotherapy and felt additional support was needed. ${ }^{2}$

Objectives: Using multiple validated instruments, this study aims to quantify the psychosocial impact of UCTD.

Methods: The Hospital for Special Surgery UCTD and Overlap Registry includes UCTD patients aged $\geq 18$ years with ANA $\geq 1: 80$ and $\geq 1$ sign or symptom of rheumatic disease who do not fulfill classification criteria for a defined CTD. We administered the 36-Item Short Form Health Survey (SF36), General Anxiety Disorder-7 (GAD-7), Beck Depression Inventory (BDI), and Fatigue Severity Scale (FSS) to all patients to assess HRQoL, anxiety, depression, and fatigue. Instruments were scored based on established algorithms and results were summarized using predefined scales and severity thresholds.

Results: The composite questionnaire was administered to 85 UCTD patients and completed by $75(97.3 \%$ female, $60 \%$ white, mean age \pm SD $48.8 \pm 13.6$ years). The SF-36 Physical Component Summary mean score was 37.8 and Mental Component Summary mean score was 41.1. Across the 8 SF-36 subscales, mean scores were lowest for role limitations due to physical health (39.3) and vitality (39.7) and highest for physical functioning (67.2), role limitations due to emotional health (67.1), and mental health (67.1). Approximately half of UCTD patients reported anxiety (GAD-7 $\geq 6$ ); $20 \%$ had moderate/severe anxiety (GAD-7 $\geq 10$ ). The prevalence of depression $(\mathrm{BDI} \geq 14$ ) was $26.7 \% ; 13.3 \%$ had moderate/severe depression (BDI $\geq$ $20)$. Fatigue (FSS $\geq 3$ ) was reported by $82.8 \%$ of patients (median FSS score of 4.7) [Table 1].
Table 1. Psychosocial Survey Scores of Patients with Undifferentiated Connective Tissue Disease $(n=75)$

\begin{tabular}{|c|c|c|}
\hline \multirow{3}{*}{$\begin{array}{l}\text { 36-Item Short Form Health Survey } \\
\text { (SF-36) }\end{array}$} & Physical Component Summary & $38.2(11.2)$ \\
\hline & Physical functioning & $67.2(26.3)$ \\
\hline & •Role-Physical & $39.3(46.3)$ \\
\hline \multirow[t]{7}{*}{ Range $1-100-$ Mean $(S D)^{*}$} & •Bodily Pain & $49.5(22.1)$ \\
\hline & oGeneral Health & $42.9(21.5)$ \\
\hline & Mental Component Summary & $41.3(10.7)$ \\
\hline & -Vitality & $39.7(21.7)$ \\
\hline & -Social Functioning & $59.3(25.9)$ \\
\hline & 'Role-Emotional & $67.1(41.9)$ \\
\hline & oMental Health & $67.1(18.3)$ \\
\hline \multirow{3}{*}{$\begin{array}{l}\text { Generalized Anxiety Disorder-7 } \\
\text { (GAD-7) }\end{array}$} & None $[0-5]$ & $38(50.7)$ \\
\hline & Mild [6-10] & $22(29.3)$ \\
\hline & Moderate [11-15] & $14(18.7)$ \\
\hline Range $0-21-N(\%)^{\star *}$ & Severe [16-21] & $1(1.3)$ \\
\hline \multirow[t]{2}{*}{ Beck Depression Inventory (BDI) } & Minimal [0-13] & 55 (73.3) \\
\hline & Mild [14-19] & $10(13.3)$ \\
\hline \multirow[t]{2}{*}{ Range $0-63-N(\%)^{\star *}$} & Moderate [20-28] & $7(9.3)$ \\
\hline & Severe [29-63] & $3(4.0)$ \\
\hline \multicolumn{2}{|c|}{ Fatigue Severity Scale (FSS) Range 1-7 - Median (IQR) ${ }^{\star *}$} & $4.7(1.5)$ \\
\hline
\end{tabular}

*Higher number indicates better health state. **Higher number indicates greater severity.

Conclusion: UCTD patients have significantly impaired HRQoL and a high prevalence of anxiety, depression, and fatigue, suggesting substantial psychosocial impact of UCTD comparable to that reported in SLE. ${ }^{3,4}$ Impaired HRQoL in UCTD is driven to similar degrees by aspects of physical and mental health. In future studies, we will compare age- and sex- matched UCTD to SLE patients and longitudinally evaluate psychosocial metrics alongside clinical trajectories. REFERENCES:

[1] Dietz B, Katz P, Dall'Era M, et al. Major depression and adverse patient-reported outcomes in systemic lupus erythematosus: Results from a prospective longitudinal cohort. Arthritis Care Res. 2021;73(1):48-54.

[2] Siegel $\mathrm{CH}$, Kleinman J, Barbhaiya $\mathrm{M}$, et al. The psychosocial impact of undifferentiated connective tissue disease on patient health and well-being: A qualitative study. J Clin Rheumatol. In press.

[3] Gu M, Cheng Q, Wang X, et al. The impact of SLE on health-related quality of life assessed with SF-36: A systemic review and meta-analysis. Lupus. 2019;28(3):371-382.

[4] Zhang L, Fu T, Yin R, Zhang Q, Shen B. Prevalence of depression and anxiety in systemic lupus erythematosus: A systematic review and meta-analysis. BMC Psychiatry. 2017;17(1).

Acknowledgements: This project was supported by the Barbara Volcker Center for Women and Rheumatic Diseases and the Robin J. Sillau Memorial Research Fund for Connective Tissue Disease. Dr. Barbhaiya is supported by the Rheumatology Research Foundation Investigator Award.

Disclosure of Interests: None declared

DOI: 10.1136/annrheumdis-2021-eular.929

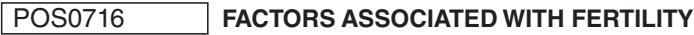 OUTCOMES IN WOMEN WITH SYSTEMIC LUPUS ERYTHEMATOSUS: A SYSTEMATIC REVIEW}

S. Giambalvo ${ }^{1}$, C. Garaffoni ${ }^{1}$, E. Silvagni ${ }^{1}$, F. Furini ${ }^{2}$, M. Govoni ${ }^{1}$, A. Bortoluzzi ${ }^{1}$. ${ }^{1}$ Unit of Rheumatology, Azienda Ospedaliera-Universitaria Sant'Anna, Department of Medical Sciences, University of Ferrara, Ferrara, Italy; ${ }^{2}$ Unit of Rheumatology, Ospedale Maggiore, Bologna, Italy

Background: Fertility is thought to be not reduced in women affected by systemic lupus erythematosus (SLE), however disease-related factors, psychosocial effects of chronic disease as well as medication exposure might impair gonadal function.

Objectives: The aim of this systematic review was to explore clinical, hormonal, serological, instrumental and management factors associated with fertility outcomes in women of childbearing age with SLE.

Methods: This systematic review was conducted following the Preferred Reporting Items for systematic reviews and Meta-analysis (PRISMA) statement. All articles available in English, published from 1972 to 15th August 2020 in Pubmed, EMBASE, Scopus and Cochrane Library. Study selection and data collection were performed by two independent reviewers. All data were extracted using a standardized template. Risk of bias of the included studies was assessed by using the NIH risk-of-bias tool [1]. 